SELECCIONES MATEMÁTICAS
Universidad Nacional de Trujillo
ISSN: $2411-1783$ (Online)
Vol. 06(01): $35-40(2019)$

\title{
Una Extensión de la Desigualdad de John-Nirenberg.
}

\section{An Extension of the Inequality of John-Nirenberg.}

Alejandro Ortiz Fernández *

Received, Jan. 23, 2019

Accepted, May. 01, 2019

DOI: http://dx.doi.org/10.17268/sel.mat.2019.01.06

\section{Resumen}

El objetivo de esta nota es presentar una extensión de la desigualdad de John-Nirenberg relativo a una caracterización de los espacios de oscilación media acotada (espacios BMO). Se menciona otra extensión de esta desigualdad.

Palabras clave. John-Nirenberg, BMO, Calderón-Zygmund, función $\varphi$, caracterización.

\section{Abstract}

The objetive of this note is to present an extension of the inequality of John-Nirenberg relative to a characterization of the spaces of bounded mean oscillation (spaces BMO). Another extension of this inequality is mentioned.

Keywords. John-Nirenberg, BMO, Calderón-Zygmund, function $\varphi$, characterization.

1. Los Espacios BMO. Desigualdad de John-Nirenberg.. Los espacios de oscilación media acotadas, espacios BMO, fueron introducidos por F. John- L. Nirenberg en 1961 [1] ; estos espacios fueron, y son, importantes en el desarrollo del análisis armónico. Por definición,

$$
B M O=\left\{f \in L^{1}\left(Q_{o}\right) /[f]_{B M O} \equiv \sup _{Q \subset Q_{o}} \frac{1}{|Q|} \int_{Q}\left|f(x)-f_{Q}\right| d x \leq M<\infty\right\}
$$

donde $Q_{o}$ es un cubo fijo en $R^{n}, Q_{o}, Q$ son cubos con lados paralelos a los ejes coordenados, $f_{Q}=\frac{1}{|Q|} \int_{Q} f ;|Q|$ medida de Lebesgue de $Q$.

Con la norma $\|f\|_{B M O}=\|f\|_{L^{1}\left(Q_{o}\right)}+[f]_{B M O}$, BMO es un espacio de Banach. Remarcamos que en la definición de BMO, en vez del promedio $f_{Q}$ se podría usar constantes o polinomios.

Proposición 1. $L^{\infty} \subset B M O$ (toda función acotada en $Q_{o}$ está en BMO) con inclusión estricta pues se tiene $\log |x| \in B M O$. (Ver [2], pag.89).

Se tiene el siguiente argumento. "Sea $f \in L^{1}(Q)$ tal que para todo cubo $Q$ se le asocia un número real $c_{Q}$ tal que el conjunto $E_{\alpha}=\left\{x \in Q /\left|f(x)-c_{Q}\right|>\alpha\right\}$ satisface

$$
w(\alpha)=\left|E_{\alpha}\right| \leq A e^{-b \alpha}|Q|
$$

donde $\alpha>0$ real; $A, b$ son apropiadas constantes. Entonces $f \in B M O$."

La prueba del argumento (*) descansa en el siguiente resultado.

\footnotetext{
*Sección Matemática. Pontificia Universidad Católica del Perú. (jortiz@pucp.edu.pe) 
Lema 1. Sea $g(t), t \geq 0$, una función continuamente diferenciable en $[0, \infty)$ tal que $g(0)=0$, entonces $\int_{Q} g(|f(x)-c|) d x=\int_{o}^{\infty} w(\alpha) d g(\alpha)$.

Prueba de (*). Si $g(t)=t, \int_{Q}\left|f(x)-c_{Q}\right| d x=\int_{o}^{\infty} w(\alpha) d(\alpha)$

$$
\leq A|Q| \int_{o}^{\infty} e^{-b \alpha} d \alpha=\frac{A}{\alpha}|Q| \text {. Luego, } f \in B M O \text {. }
$$

La parte crucial es el reciproco de este resultado, es el teorema de John-Nirenberg.

Teorema 1 (J-N). . "Si $f \in B M O$, entonces $w(\alpha) \leqslant A e^{-b \alpha\|f\|_{B M O}^{-1}\left|Q_{o}\right|}$, donde $\alpha>0 ; A, b>0$ son apropiadas constantes que dependen de $n$."

(Para la prueba de este resultado ver, por ejemplo, [2], pag. 92).

2. Espacios $B M O_{\varphi}$.. Sea $\varphi(t)$ una función positiva, no-decreciente, definida sobre $R^{+}$. Por definición,

$$
B M O_{\varphi}=\left\{f \in L^{1}\left(Q_{o}\right) /[f]_{B M O_{\varphi}} \equiv \sup _{Q \subset Q_{o}} \frac{1}{\varphi(|Q|)} \frac{1}{|Q|} \int_{Q}\left|f(x)-C_{Q}\right| d x \leq M<\infty\right\}
$$

donde los cubos son asumidos de medida finita y lados paralelos a los ejes coordenados. Podemos usar $f_{Q}$ en vez de la constante $C_{Q}$. Estos espacios $B M O_{\varphi}$ fueron inicialmente considerados por S. Spanne en 1965. (Ver [4]). Se verifica que formas particulares de $\varphi$ hacen coincidir isomorficamente $B M O_{\varphi}$ con algunos clásicos espacios de funciones. Veamos,

- si $\varphi(t)=1, B M O_{\varphi}=B M O$;

- si $\varphi(t)=t^{\alpha}, 0<\alpha<1$, entonces $B M O_{\varphi}=\bigwedge_{\alpha}$, donde $\bigwedge_{\alpha}$ es el espacio de Lipschitz

$\bigwedge_{\alpha}=\left\{f \in L^{\infty} /\|f\|_{\bigwedge_{\alpha}}=\sup _{x, y} \frac{|f(x)-f(y)|}{|x-y|^{\alpha}}<\infty\right\}$

Si $-1<\alpha<0$, entonces $B M O_{\varphi}=L^{p, \lambda}$, con $\alpha=-\frac{\lambda}{p}$, donde $L^{p, \lambda}$ es el espacio de Morrey

$L^{p, \lambda}=\left\{f \in L^{1}\left(Q_{o}\right) /\|f\|_{L^{p, \lambda}}=\sup _{Q}\left\{\frac{1}{r^{\lambda}} \int_{Q}|f(x)|^{p} d x\right\}^{1 / p}\right\}<\infty$,

donde $r$ es la longitud del lado de $Q, 1 \leq p<\infty$.

Si $\lim _{r \longrightarrow 0} \varphi(r)=a \neq 0$, entonces $L^{\infty} \subset B M O_{\varphi}$.

\section{Extensión de la Desigualdad de John-Nirenberg. La parte inmediata es el}

Lema 2. Sea $f \in L^{1}(Q)$, $\alpha>0$ real, tal que para todo $Q \subset Q_{o}$ se le asocia un número $c_{Q}$ tal que $E_{\alpha}=\left\{x \in Q /\left|f(x)-c_{Q}\right|>\alpha\right\}$ satisface

$w(\alpha)=\left|E_{\alpha}\right| \leq A e^{-b \alpha} \varphi\left(2^{-k n}|Q|\right)|Q|$,

con $k \in Z^{+}, A, b$ constantes positivas. Entonces, $f \in B M O_{\varphi}$.

Prueba.

$$
\begin{aligned}
& \int_{Q}\left|f(x)-c_{Q}\right| d x=\int_{o}^{\infty} w(\alpha) d \alpha \leq A \varphi\left(2^{-k n|Q|}\right) \int_{o}^{\infty} e^{-b \alpha} d x|Q| \\
& \leq A_{1} \varphi(|Q|)|Q| .
\end{aligned}
$$

La prueba del recíproco de este lema 2 es el objetivo de esta nota.

Teorema $2(\varphi)$. Si $f \in B M O_{\varphi}$ y $\alpha>0$ real, entonces

$$
w(\alpha)=\left|\left\{x \in Q_{o} /\left|f(x)-f_{Q_{o}}\right|>\alpha\right\}\right| \leq A e^{-b \alpha\|f\|_{B M O_{\varphi}}^{-1}} \cdot \varphi\left(2^{-k n}\left|Q_{o}\right|\right)\left|Q_{o}\right|
$$


donde $A=c A_{1} \operatorname{con} A_{1}=2^{2^{\frac{n}{n+1}+n}}, c>0$ (independiente de $k$ ), $b=\frac{n}{2^{n+1}} \log 2, k=\left[\frac{\alpha-1}{2^{n+1}}\right]$ (parte entera).

Previamente veamos algunos convenios como asumir que $\|f\|_{B M O_{\varphi}}=1$. Así mismo, observemos:

de

$$
\frac{1}{\varphi(|Q|)} \frac{1}{|Q|} \int_{Q}\left|f(x)-f_{Q}\right| d x \leq M<\infty
$$

ó

$$
\frac{1}{\varphi(|Q|)} \frac{1}{|Q|} \int_{Q}\left|\frac{f(x)-f_{Q}}{M}\right| d x \leq 1
$$

se puede asumir que, redefiniendo $f$ en $B M O_{\varphi}, f_{Q}=0$ y $M=1$. Por otro lado, si $\left|Q_{o}\right|=a \neq 1$ y si $d$ es la longitud del lado de $Q_{o}$, tendremos $\left(\frac{d}{b}\right)^{n}=1 \operatorname{con} b=\sqrt[n]{a}$, y se puede asumir que $\left|Q_{o}\right|=1$; también se puede asumir que $\varphi\left(\left|Q_{o}\right|\right)=\varphi(1)=1$, pues si $\varphi(1)=c \neq 1, \varphi_{1}(t)=\frac{\varphi(t)}{c}$ tiene las mismas características que $\varphi$.

La prueba del teorema se hará por etapas en donde es fundamental el lema de Calderón-Zygmund.

Lema 3. (Descomposición de Calderón-Zygmund). Sea $f$ una función integrable definida en un cubo $Q_{o} y$ sea $\lambda>0$ real tal que $\frac{1}{\left|Q_{o}\right|} \int_{Q_{o}}|f(x)| d x \leq \lambda$. Entonces existe una familia enumerable $\left\{Q_{k}\right\}$ de cubos abiertos disjuntos en $Q_{o}$ tal que:

(a). $|f(x)| \leq \lambda$ c.t.p. si $x \in Q_{o}-\bigcup_{k} Q_{k}$;

(b) $\lambda<\frac{1}{\left|Q_{k}\right|} \int_{Q_{k}}|f(x)| d x \leq 2^{n} \lambda$;

(c). $\sum_{k}\left|Q_{k}\right|<\frac{1}{\lambda} \int_{Q_{o}}|f(x)| d x$.

Esta fundamental descomposición fue introducida por Calderón-Zygmund en 1952 en un fundamental trabajo sobre integrales singulares. Por razones didácticas ofrecemos su demostración. Veamos. Dividamos $Q_{o}$ en $2^{n}$ cubos abiertos congruentes (dividiendo por 2 sus lados). Con estos subcubos se presentan dos casos:

(I). $\frac{1}{\left|Q_{i}^{\prime}\right|} \int_{Q_{i}^{\prime}}|f(x)| d x>\lambda$; (II). $\frac{1}{\left|Q_{i}^{\prime \prime}\right|} \int_{Q_{i}^{\prime \prime}}|f(x)| d x \leq \lambda$.

Conservemos los cubos $Q_{i}^{\prime}$ que satisfacen (I) (que es parte de (b)), mientras que los cubos $Q_{i}^{\prime \prime}$, que satisfacen (II), son sometidos al anterior proceso, esto es, son divididos en $2^{n}$ nuevos subcubos congruentes, dando origen a una nueva generación de cubos en donde nuevamente tenemos

(I). $\frac{1}{\left|Q_{i i}^{\prime}\right|} \int_{Q_{i i}^{\prime}}|f(x)| d x>\lambda ;$ (II). $\frac{1}{\left|Q_{i i}^{\prime \prime}\right|} \int_{Q_{i i}^{\prime \prime}}|f(x)| d x \leq \lambda$.

Retenemos los cubos $Q_{i i}^{\prime}$, mientras $\operatorname{los} Q_{i i}^{\prime \prime}$ son sometidos otra vez al anterior proceso. Y así sucesivamente ... De esta manera, renumerando obtenemos familias de cubos abiertos disjuntos, de distintas generaciones $Q_{1}, Q_{2}, \ldots, Q_{k}, \ldots$ tal que

$$
\lambda\left|Q_{k}\right|<\int_{Q_{k}}|f(x)| d x \leq 2^{n} \lambda\left|Q_{k}\right| \text { lo que implica (b). }
$$

Por otro lado, $\left|Q_{k}\right|<\frac{1}{\lambda} \int_{Q_{k}}|f(x)| d x$, de donde $\sum_{k}\left|Q_{k}\right|<\frac{1}{\lambda} \int_{Q_{o}}|f(x)| d x$, que es (c).

Finalmente, sea $x \in Q_{o}-\bigcup_{k} Q_{k}$, esto es, $x$ pertenece a algún cubo del tipo $Q_{k}^{\prime \prime}$, donde (por construcción) $\left|Q_{k}^{\prime \prime}\right| \longrightarrow 0$ cuando $k \longrightarrow \infty$. Como tenemos

$\frac{1}{\left|Q_{k}^{\prime \prime}\right|} \int_{Q_{k}^{\prime \prime}}|f(y)| d y \leq \lambda$ 
por el teorema de diferenciación de Lebesgue se tiene

$|f(x)|=\lim _{k \longrightarrow \infty} \frac{1}{\left|Q_{k}^{\prime \prime}\right|} \int_{Q_{k}^{\prime \prime}}|f(y)| d y \leq \lambda$, c.t.p. Esto es (a)..

En la ruta hacia el teorema $-\varphi$ se tiene el

Lema 4. Sea $\alpha>0$ un real dado y $f \in \mathrm{BMO}_{\varphi}$. Si $\frac{1}{\left|Q_{o}\right|} \int_{Q_{o}}|f(x)| d x<\alpha$,

entonces se tiene $\alpha<\frac{1}{|C|} \int_{C}|f(x)| d x \leq \alpha+2^{n} \varphi\left(2^{n}|C|\right)$

donde $C \in D_{\alpha}$, siendo $D_{\alpha}$ la unión de los cubos diádicos disjuntos que aparecen en el lema 3.

Prueba. Por el lema 3 se tiene $\alpha<\frac{1}{|C|} \int_{C}|f(x)| d x \leq 2^{n} \alpha, \forall C \in D_{\alpha}$.

Desde que por hipótesis se tiene $\frac{1}{\left|Q_{o}\right|} \int_{Q_{o}}|f(x)| d x<\alpha$, entonces $C \neq Q_{o}$. Luego, $C$ fue obtenido a partir de una descomposición diádica de un cubo más grande, que llamaremos $C_{o}$, el cual satisface

$\frac{1}{\left|C_{o}\right|} \int_{C_{o}}|f(x)| d x \leq \alpha$, (esto es, $\left|f_{C_{o}}\right| \leq \alpha$. Entonces,

$\frac{1}{|C|} \int_{C}|f(x)| d x \leq \frac{1}{|C|} \int_{C}\left|f(x)-f_{C_{o}}\right| d x+\left|f_{C_{o}}\right| \leq 2^{n} \varphi\left(2^{n}|C|\right)\|f\|_{B M O_{\varphi}}+\alpha$.

Nota. De $2^{n}|C| \leq\left|Q_{o}\right|=1$ se observa que $\varphi\left(2^{n}|C|\right) \leq 1$, luego se obtiene $\alpha<\frac{1}{|C|} \int_{C}|f(x)| d x \leq \alpha+2^{n}$, un resultado debido a U. Neri (1976).

Lema 5. Si $\tilde{\alpha}=\alpha+2^{n+1}$, entonces $D_{\tilde{\alpha}} \subset D_{\alpha}$ y se tiene

$$
\left|D_{\tilde{\alpha}}\right| \leq 2^{-n}\left|D_{\alpha}\right| \varphi\left(\left|D_{\alpha}\right|\right)
$$

Prueba. En general, del lema 3 se obtiene: si $\alpha<\alpha^{\prime}$ entonces $D_{\alpha^{\prime}} \subset D_{\alpha}$. Por otro si $C \in D_{\alpha}$ es arbitrario, entonces por la nota se tiene

$$
\frac{1}{|C|} \int_{C}|f(x)| d x \leq \alpha+2^{n} \leq \tilde{\alpha}
$$

lo que significa que $C$ fue subdividido en la descomposición correspondiente a $D_{\tilde{\alpha}}$.

Pongamos $D^{\prime}=D_{\tilde{\alpha}} \bigcap C$.

Ahora, un argumento geométrico en el lema 3 permite ver que $D^{\prime}=\phi$ ó que $D^{\prime}$ es la unión de cubos disjuntos en $D_{\tilde{\alpha}}$.

Además se tiene,

$$
\begin{aligned}
& \tilde{\alpha}<\frac{1}{\left|D^{\prime}\right|} \int_{D^{\prime}}|f(x)| d x \leq \frac{1}{\left|D^{\prime}\right|} \int_{D^{\prime}}\left|f(x)-f_{C}\right| d x+\frac{1}{\left|D^{\prime}\right|}\left|D^{\prime}\right|\left|f_{C}\right| \\
\leq & \frac{1}{\left|D^{\prime}\right|} \int_{C}\left|f(x)-f_{C}\right| d x+\alpha+2^{n} \leq \frac{|C| \varphi(|C|)}{\left|D^{\prime}\right|}+\alpha+2^{n} .
\end{aligned}
$$

Es decir, se tiene

$\alpha+2^{n+1} \leq \frac{|C| \varphi(|C|)}{\left|D^{\prime}\right|}+\alpha+2^{n}$, esto es,

$\left|D^{\prime}\right| \leq 2^{-n}|C| \varphi(|C|)$. Luego,

$$
\begin{gathered}
\left|D_{\tilde{\alpha}}\right|=\sum\left|D^{\prime}\right| \leq 2^{-n} \sum_{\substack{C \in D_{\alpha} \\
\leq 2^{-n} \varphi\left(\left|D_{\alpha}\right|\right)\left|D_{\alpha}\right| . .}}|C| \varphi(|C|) \leq 2^{-n} \varphi\left(\left|D_{\alpha}\right|\right) \sum_{C \in D_{\alpha}}|C| \\
{[\alpha, 1]}
\end{gathered}
$$

Prueba del Teorema $-\varphi$. Pongamos $k=\left[\frac{\alpha-1}{2^{n+1}}\right]$; entonces, si $\alpha_{1}=1+k 2^{n+1}$ se tiene $1 \leq \alpha_{1} \leq \alpha$, de donde $w(\alpha)=\left|E_{\alpha}\right| \leq\left|E_{\alpha_{1}}\right|=($ lema 3$)=\left|D_{\alpha_{1}}\right|=\left|D_{1+k 2^{n+1}}\right|$. 
Aplicando reiteradamente el lema 5, luego de $k$ pasos, se obtiene

$\left|D_{1+k 2^{n+1}}\right| \leq 2^{-n}\left|D_{1+(k-1) 2^{n+1}}\right| \varphi\left(\left|D_{1+(k-1) 2^{n+1}}\right|\right) \leq$

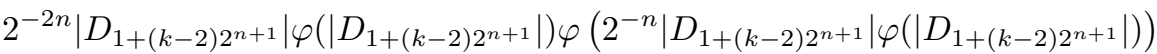

$\leq$ (desde que $\varphi$ es no-decreciente y $\left.\varphi\left(\left|D_{1+(k-2) 2^{n+1}}\right|\right)<\varphi\left(\left|Q_{o}\right|\right)=1\right)$

$\leq 2^{-2 n}\left|D_{1+(k-2) 2^{n+1}}\right| \varphi\left(2^{-n}\left|D_{1+(k-2) 2^{n+1}}\right|\right) \leq \ldots \leq c 2^{-k n}\left|D_{1}\right| \varphi\left(2^{-k n}\left|D_{1}\right|\right)$

$\leq c 2^{-k n} \varphi\left(2^{-k n}\left|Q_{o}\right|\right)\left|Q_{o}\right|$.

En conclusión se ha obtenido $w(\alpha) \leq c 2^{-k n} \varphi\left(2^{-k n}\left|Q_{o}\right|\right)\left|Q_{o}\right|$.

Por otro lado, se sabe que

$\frac{\alpha-1}{2^{n+1}} \leq k+1$, luego $-n k \leq-n\left(\frac{\alpha-1}{2^{n+1}}-1\right)$, de donde $2^{-k n} \leq 2^{-n\left(\frac{\alpha-1}{2^{n+1}}-1\right)}$.

Y así finalmente se tiene

$$
w(\alpha) \leq c 2^{\frac{n}{2^{n+1}+n}} \cdot 2^{-\alpha \cdot \frac{n}{2^{n+1}}} \varphi\left(2^{-k n}\left|Q_{o}\right|\right)\left|Q_{o}\right|
$$

la cual es la tesis

$$
w(\alpha) \leq A e^{-b \alpha\|f\|_{B M O_{\alpha}}^{-1} \cdot \varphi\left(2^{-k n}\left|Q_{o}\right|\right)\left|Q_{o}\right|}
$$

con

$$
A=c A_{1}, \quad A_{1}=2^{\frac{n}{2^{n+1}}+n}, \quad b=\frac{n}{2^{n+1}} \log 2 .
$$

4. Una $\Psi$ - extensión. . En [6], A. Torchinsky ha considerado a los espacios $B M O_{\varphi}$ (pag.220; 6.20) y posteriormente obtuvo una versión de la desigualdad de John-Nirenberg generalizada; algunos detalles y casos particulares de esta versión puede verse en [3] , pag.153. Brevemente veamos algunos argumentos. Sea $\varphi(t)$ una función continua, no-decreciente, de valor real tal que $\varphi(0)=0 . B M O_{\varphi}$ es definido como en la sección 2 siendo la seminorma

$$
\|f\|_{B M O_{\alpha}} \equiv\|f\|_{*, \varphi}=\sup _{Q \subset Q_{o}} \frac{1}{\varphi(|Q|)} \frac{1}{|Q|} \int_{Q}\left|f(x)-f_{Q}\right| d x
$$

Torchinsky considera la función $\Psi_{\lambda}(t)=\int_{t}^{2^{n} \lambda} \varphi(y) \frac{d y}{y}$ y obtiene el

Teorema $3(\Psi)$. Sea $f \in B M O_{\varphi}$. Si $\lim _{t \rightarrow o^{+}} \Psi_{\lambda}(t)=+\infty$, entonces existe una constante c tal que para todo $Q \subset Q_{o}$ se tiene

$$
w(\alpha) \equiv\left|\left\{x \in Q /\left|f(x)-f_{Q}\right|>\alpha\right\}\right|<c \Psi_{|Q|}^{-1}\left(\frac{\alpha}{\|f\|_{*, \varphi}}\right) .
$$

En consecuencia se tiene la proposición

Proposición 2. Sea $\varphi(t)=\eta\left(\log \left(\frac{1}{t}\right)\right)$, donde $\eta(t)=\Phi^{\prime}(t)$ siendo $\Phi(t)$ una función derivable, no-decreciente $\operatorname{con} \Phi(0)=0 . S i$

$$
\Psi_{|Q|}(t)=\int_{t}^{2^{n}|Q|} \eta\left(\log \left(\frac{1}{y}\right)\right) \frac{d y}{y}
$$

entonces se tiene $\Psi_{|Q|}^{-1}(t) \leq c e^{-\frac{1}{2} \Phi^{-1}(t)} \cdot|Q|$.

Corolario 1. (Recuperación de la desigualdad de John-Nirenberg para BMO). Sea $\eta(y)=1$ y $\varphi(t)=1$; como $\Phi$ es tal que $\Phi^{\prime}(t)=1$ se tiene que $\Phi(t)=t$, esto es, $\Phi^{-1}(t)=t$. Entonces,

$\Psi_{|Q|}^{-1}(t) \leq c e^{-\frac{1}{2} \Phi^{-1}(t)} \cdot|Q|=c e^{-\frac{1}{2} t} \cdot|Q|$,

y por el teorema $-\Psi$ tenemos 
$w(\alpha) \leq c \Psi_{|Q|}^{-1}\left(\frac{\alpha}{\|f\|_{*, \varphi}}\right) \leq c e^{-\frac{1}{2} \alpha\|f\|_{*, \varphi}^{-1}} \cdot|Q|$,

que es la desigualdad de John-Nirenberg para BMO..

Agradecimiento. El autor expresa su agradecimiento al Prof. Alberto Torchinsky, quien propuso la extensión mencionada y nos guió en la elaboración de esta nota $([5])$ pero asumimos la entera responsabilidad de la misma.

\section{Referencias}

[1] John, F.-Nirenberg, L: “On Functions of Bounded Mean Oscillation” Comm. P. Appl. Math. 1961.

[2] Ortiz, Alejandro: "Tópicos sobre análisis armónico" Dpto. Matemática. UNT. Perú. 1988.

[3] Ortiz, Alejandro: "Casos particulares de la desigualdad de John-Nirenberg para espacios BMO " PRO MATHEMATICA. Vol.IV Nos. 7-8. PUCP. 1990.

[4] Spanne, S.: "Some Fuction Spaces Defined Using the Mean Oscillation over Cubes" Ann. Sup. Pisa. 1965

[5] Torchinsky, Alberto: (comunicación personal. 1984)

[6] Torchinsky, Alberto: "Real-Variable Methods in Harmonic Analysis" Academic Press. 1986 